\title{
ON A CERTAIN TYPE OF HOMOGENEOUS PLANE CONTINUUM
}

F. BURTON JONES

In 1920 very little was known about the class of homogeneous, bounded continua in the plane. At that time Knaster and Kuratowski [1] raised the question: ${ }^{1}$ Is every such (nondegenerate) continuum a simple closed curve? Mazurkiewicz [2] showed such a continuum is a simple closed curve if it is locally connected, and I showed this is the case if the continuum is aposyndetic [3]. H. J. Cohen [4] proved that if a homogeneous, bounded, plane continuum contains a simple closed curve, it is a simple closed curve. And finally I proved that every homogeneous, compact continuum lying in but not separating a plane is indecomposable [5].

So the class of homogeneous, bounded, plane continua may be typed as follows:

Type 1. Those which do not separate the plane. (These must all be indecomposable, and continua of Type 1 other than degenerate ones are known to exist [6 and 7].)

Type 2. Those which are decomposable. (These must all separate the plane, and continua of Type 2 other than simple closed curves are known to exist [8].)

Type 3. Those which separate the plane but are indecomposable. (Whether any of this type exists is not known. However, see [9, Example 2, pp. 48-49].)

It is the purpose of this paper to show that each homogeneous, bounded, plane continuum of Type 2 is either a simple closed curve or becomes one under a natural aposyndetic decomposition, ${ }^{2}$ the elements of the decomposition being mutually homeomorphic continua of Type 1. In other words, thinking of a plane as an upper semicontinuous collection of continua (each lying in but not separating a given plane), every continuum of Type 2 is the sum of the elements of a simple closed curve lying in a plane of elements of Type 1.

Presented to the Society, September 2, 1954; received by the editors November 19, 1954.

${ }^{1}$ Numbers in brackets refer to the bibliography at the end of this paper.

${ }^{2}$ A continuum $M$ is said to be aposyndetic provided that if $x$ and $y$ are distinct points of $M$, there exist a continuum $K$ and an open (rel. $M$ ) set $U$ such that $M-y \supset K \supset U \supset x$.

An aposyndetic decomposition [10] is an upper semi-continuous collection of mutually exclusive continua filling up the given continuum and which with respect to its elements (as points) is an aposyndetic continuum. 
It is convenient to establish the following more general decomposition theorem for homogeneous, compact continua lying in a metric space. (A compact continuum lying in a regular, semi-metric space or in a Moore space is itself a metric space.)

Notation. If $x$ is a point of a continuum $M$, then $L_{x}$ denotes $x$ together with all points $y$ of $M$ such that $M$ is not aposyndetic at $y$ with respect to $x$ and $U_{x}$ denotes $M-L_{x}$.

Theorem 1. Suppose that $M$ is a decomposable, homogeneous, compact, metric continuum. Then there exists a nondegenerate continuous collection $G$ of mutually exclusive continua filling up $M$ such that (a) with respect to its elements (as points) $G$ is a homogeneous, aposyndetic, compact, metric continuum, (b) if $x$ is a point of $M$, then $L_{x}$ is an element of $G$, and (c) if $g$ is an element of $G$ and $K$ is a subcontinuum of $M$ which contains both a point of $g$ and a point of $M-g$, then $g$ is a subset of $K$.

Proof. If for some point $x$ of $M, L_{x}$ is degenerate, then all elements of $G$ are degenerate and $M$ is aposyndetic. In this case, Theorem 1 is obvious. So from here on, in this proof, it will be assumed that no element of $G$ is degenerate. It follows at once that (1) $M$ is totally nonaposyndetic.

Being totally nonaposyndetic, $M$ contains a cut point [11, p. 409]. Hence (by the homogeneity) (2) every point of $M$ is a cut point of $M .^{3}$

By [12, p. 501] some point of $M$ does not cut any two (rel.) open subsets of $M$ from each other. Again by the homogeneity of $M$, (3) no point of $M$ cuts two (rel.) open subsets of $M$ from each other.

Now for each point $p$ of $M, U_{p}$ is the set of all points of $M$ at which $M$ is aposyndetic with respect to $p$. Since $M$ is decomposable and homogeneous, (4) for each point $p$ of $M, U_{p}$ is a nondegenerate, (rel.) open subset of $M$. It follows from (3) and the definition of $U_{p}$ that (5) $p$ does not cut any two points of $U_{p}$ from each other.

Since by (2) if $p$ is a point of $M, p$ cuts some two points from each other, it follows from (5) that (6) $p$ cuts some point o from every point of $U_{p}$. And from the homogeneity it is true that (7) for each point $o$ of $M$, there exists a point $p$ of $M$ such that $p$ cuts $o$ from every point of $U_{p}$. In this case every point of $U_{p}$ is a point of $U_{o}$. Hence by the lemma of [5], (8) $U_{p}=U_{o}$.

Suppose that $U$ is an open subset of $M$ and that $H$ is a subset of $M-U$ such that in order for a point $x$ of $M$ to belong to $H$ it is necessary and sufficient that $U_{x}=U$. It follows from (4), (7) and (8) that

${ }^{3}$ A cut point of $M$ is a point whose complement in $M$ is not strongly (continuumwise) connected. As used here a cut point need not be a separating point. 
such sets $U$ and $H$ do exist and are nondegenerate. From (5) and (8) it follows that (9) if $w$ is a point of $M$ which is cut (in $M$ ) from a point of $U$ by a point of $H$, then $w$ is a point of $H$.

It is rather easy to see that $H$ is closed. Suppose that there exists a point $y$ of $\bar{H}-H$. Let $z$ be a point of $U_{y}$. Then $M$ is aposyndetic at $z$ with respect to $y$ and hence $M$ is aposyndetic at $z$ with respect to some point of $H$. Consequently $z$ belongs to $U$. But by the lemma of [5], $U_{y}$ cannot be a proper subset of $U$ and hence $U_{y}=U$ and $y$ belongs to $H$. So (10) $H$ is closed.

For each point $o$ of $H$, let $N_{o}$ denote $o$ together with all points $x$ of $H$ such that $x$ cuts $o$ from $U$. The set $N_{o}$ is closed. Suppose that for each point $o$ of $H, o$ does not cut all other points of $N_{o}$ from $U$. Now let $o_{1}$ be such a point of $H$. Then $N_{o_{1}}$ contains a point $o_{2}$ such that $N_{o_{2}}$ is a subset of $N_{o_{1}}-o_{1} ; N_{o_{2}}$ contains a point $o_{3}$ such that $N_{o_{3}}$ is a subset of $N_{o_{2}}-o_{2}$; and so on for a countably infinite number of times. Let $o_{\omega}$ denote a point of $\prod N_{o_{i}}$. Then $N_{o_{\omega}}$ is a subset of each $N_{o_{i}}$, and the process may be continued. Thus one obtains an uncountable well-ordered monotone decreasing sequence of distinct closed subsets of a compact metric space. This being impossible, it follows that for some point $o$ of $H, o$ cuts all other points of $N_{o}$ from $U$. Because of the homogeneity this must be true for every point $o$ of $H$. So (11) if a point $p$ of $H$ cuts a point $o$ of $H$ from $U$ (i.e., $p$ belongs to $N_{o}$ ), then $o$ cuts $p$ from $U$.

The set $U$ is dense in $M$. (12) Let $o$ be a point of $H$. It follows from (3) and (11) that $N_{o}$ contains no (rel.) open subset of $M$. So by (10) if $\bar{U} \neq M$ there exists an (rel.) open subset $D$ of $M$ lying in $M-(\bar{U}$ $+N_{o}$ ). Since $U_{o}=U, M$ is not aposyndetic at any point of $D$ with respect to $o$. By Theorem 6 of [11] $D$ contains a point $w$ such that $o$ cuts $w$ from a point of $U$. By (9) $w$ is a point of $H$. Hence $o$ belongs to $N_{w}$. So by (11) $N_{o}$ contains $w$, which is a contradiction.

If $p$ and $o$ are points of $M$ and $p$ cuts $o$ from $U_{o}$, it follows from (4) and (12) that $p$ cuts $o$ from a point of $U_{p}$. By (5), $p$ cuts $o$ from $U_{p}$ and, by (8), $U_{p}=U_{o}$. So if $H$ is the set of all points $x$ such that $U_{x}=U_{o}$, then $p$ is a point of $H$ and hence (by the definition of $N_{o}$ ) $p$ is a point of $N_{o}$. So $N_{o}$ may be characterized without reference to $H$ as follows: $N_{o}$ is $o$ together with all points $x$ of $M$ such that $x$ cuts $o$ from $U_{o}$. Then if $p$ is a point of $N_{o}, U_{p}=U_{o}$ and, by (11), $N_{p}=N_{o .}{ }^{4}$ Hence if $o$ is a point of $M, N_{o}$ may also be characterized as follows: $N_{o}$ is $o$ together with all points $x$ of $M$ such that $o$ cuts $x$ from $U_{o}$.

Now suppose that for some point $o$ of $M, N_{o}+U_{o} \neq M$, i.e., there is a

${ }^{4}$ The reader should observe that if $x$ and $y$ are points of $M$ then either $N_{x}=N_{y}$ or $N_{x} \cdot N_{y}=0$. 
point $q$ of $M$ such that $M$ is not aposyndetic at $q$ with respect to $o$ (or any other point of $N_{o}$ ) but no point of $N_{o}$ cuts $q$ from $U_{o}$. Because of the homogeneity, every point $q$ of $M$ is so topologically related to some point $o$ of $M$. Since $M$ is decomposable, $M$ is the sum of two proper subcontinua $M_{1}$ and $M_{2}$. Let $V_{1}=M-M_{2}, V_{2}=M-M_{1}$ and $D$ be an (rel.) open subset of $M$ such that $V_{1}$ contains $\bar{D}$. For each point $q$ of $\bar{D}$, there exists a point $o$ of $M$ such that $M$ is not aposyndetic at $q$ with respect to $o$ but $q$ does not belong to $N_{o}$. Since $q$ does not cut $o$ from $U_{q}$ and $V_{2} \cdot U_{q} \neq 0, q$ does not cut $o$ from $V_{2}$. So there exists a continuum $K_{o}$ in $M-q$ irreducible from $o$ to $\bar{V}_{2}$. Now for each point $q$ of $\bar{D}$ let $d(q)$ be the largest number such that for some $o$ and $K_{o}$ (with the prescribed properties), $d(q)=d\left(K_{o}, q\right)$ and let $o(q)$ and $K_{o}(q)$ be some one definite such pair, $o$ and $K_{o}$. For each positive integer $n$, let $E_{n}$ denote the set of all points $q$ of $\bar{D}$ such that $d(q) \geqq 1 / n$. First, observe that $E_{n}$ is closed, and second, that $\sum_{n} E_{n}=\bar{D}$. Hence for some positive integer $n^{\prime}, E_{n^{\prime}}$ contains an open subset of $M$; so there exists a circular region $R_{r}(p)$ (with radius $r<1 / n^{\prime}$ and center $p$ in $E_{n^{\prime}}$ ) such that every point of $M \cdot R_{r}(p)$ belongs to $E_{n}{ }^{\prime}$. It is easy to see that if $s=1 / n^{\prime}-r$, then for each point $q$ of $M \cdot R_{r}(p) \cdot R_{s}(p)$ every point of $K_{o}(q)$ is outside of $R_{r}(p)$.

Let $M_{0}$ denote the closure of $\sum K_{o}(q)$ f for all $q$ in $\left.M \cdot R_{r}(p) \cdot R_{s}(p)\right\}$. Let $z$ be a point of $V_{2}$. By Theorem 6 of [11] there exist a point $x$ of $M \cdot R_{r}(p) \cdot R_{s}(p)$ and a point $y$ of $M_{0}$ such that $y$ cuts $x$ from $z$ in $M$. Either $y$ cuts $x$ from $U_{y}$ or $y$ cuts $z$ from $U_{y}$. Case I. Suppose that $y$ cuts $z$ from $U_{y}$; then $z$ belongs to $N_{y}$. Since $M_{0}$ is a subset of $M_{1}$, $y$ belongs to $M_{1}$. Furthermore $M_{1}$ contains $V_{1}$ which intersects $U_{y}$. So $M_{1}$ contains $y$ and a point of $U_{y}$ but does not contain $z$. This is a contradiction because $z$ cuts $y$ from $U_{y}$. Case II. Suppose that $y$ cuts $x$ from $U_{y}$, then $x$ belongs to $N_{y}$. Then $M_{2}+M_{0}$ is a cont nuum containing $y+V_{2}$ but no point of $R_{r}(p) \cdot R_{s}(p)$. Since $V_{2}$ intersects $U_{y}$, $M_{2}+M_{0}$ contains $y$ and a point of $U_{y}$ but not $x$. Hence $x$ does not cut $y$ from $U_{y}$, which is a contradiction.

It follows from the preceding paragraph that for each point $o$ of $M$, $N_{o}=M-U_{o}$. Hence $N_{o}$ consists of exactly those points at which $M$ is not aposyndetic with respect to $o$. It follows from Theorem 3 of [11] that $N_{o}$ is a continuum.

Let $G$ denote the collection of all subsets $g$ of $M$ such that for some point $o$ of $g, g$ is $L_{o}$, i.e., $o$ together with all points $y$ of $M$ such that $M$ is not aposyndetic at $y$ with respect to $o$. Because of the homogeneity of $M$ and the preceding argument, each element $g$ of $G$ has the following additional properties:

(a) for some point $o$ of $g, N_{o}=g$; 
(b) for each point $x$ of $g, U_{x}=U_{o}=U(\mathrm{~g})$;

(c) for each point $x$ of $g, N_{x}=g$;

(d) if $x, y \in g, x$ cuts $y$ from $U(g)$;

(e) $M-g=U(g)$; and

(f) if $K$ is a subcontinuum of $M$ such that $K \cdot g \neq 0 \neq K \cdot U(g)$, then $K$ contains $g$.

So $G$ is a collection of mutually exclusive, homogeneous, nondegenerate continua filling up $M$ and each two elements of $G$ are homeomorphic. Furthermore $G$ is upper semi-continuous. For if this were not the case $G$ would contain a convergence sequence of elements whose sequential limiting set would contain points $a$ and $b$ belonging to different elements of $G$. Hence $M$ is aposyndetic at $a$ with respect to $b$, i.e., there exist a subcontinuum $K$ of $M$ and an open subset $V$ of $M$ such that $M-b \supset K \supset V \supset a$. It follows that some $g$ of $G$ (not containing $a$ ) contains a point $x$ of $V$ and a point $y$ of $M-K$. This is a contradiction of (f). Since $G$ must be continuous at some one of its elements, it follows from the homogeneity that $G$ is continuous.

Theorem 2. Suppose that $M$ is a homogeneous, decomposable, bounded, plane continuum. Let $G$ denote the collection of all subsets $g$ of $M$ such that for some point $x$ of $M, g$ is $L_{x}$. Then $G$ is a continuous collection of mutually exclusive continua filling up $M$ such that

(1) with respect to its elements (as points), $G$ is a simple closed curve, and

(2) each element of $G$ is a homogeneous, bounded, plane continuum which does not separate the plane.

Proof. Suppose that $M$ is a subset of a plane $S$. It is clear that for each $g$ of $G, M-g$ is connected (in fact, by (5), $M-g$ is strongly or continuum-wise connected). Since each two elements of $g$ are homeomorphic, it follows that if one element of $G$ were to separate $S$, then each of them would separate $S$ and there would exist uncountably many mutually exclusive domains (at least one for each $g$ ) in the plane. This being impossible, no element of $G$ separates $S$. Let $G^{\prime}$ denote the upper semi-continuous collection consisting of $G$ together with each degenerate subset of $S-M$. With respect to its elements (as points), $G^{\prime}$ is a plane [13] and $G$ is a compact subcontinuum of $G^{\prime}$. Furthermore, $G$ is homogeneous and aposyndetic. Hence by [5], $G$ is a simple closed curve.

REMARKs. Because each element of $G$ in Theorem 2 is homogeneous and does not separate the plane, each such element is indecomposable. The question [5] still remains: Is every homogeneous, bounded, nondegenerate, plane continuum which does not separate the plane a 
pseudo-arc?

L. F. McAuley [10] has shown that every compact metric continuum has an aposyndetic very nearly atomic decomposition. His decomposition applied to the continuum $M$ of the theorems yields the same collection $G$ as I have obtained, but I have found no easy way to make use of it here.

Since every decomposable, homogeneous, bounded continuum in the plane must have exactly two complementary domains (this follows from Theorem 2), every homogeneous, bounded continuum in the plane having more than two complementary domains is indecomposable and of Type 3. C. E. Burgess has a somewhat stronger result than this in [14].

\section{BiBLIOGRAPHy}

1. B. Knaster and C. Kuratowski, Problème 2, Fund. Math. vol. 1 (1920) p. 223.

2. Stefan Mazurkiewicz, Sur les continua homogènes, Fund. Math. vol. 5 (1924) pp. $137-146$.

3. F. B. Jones, A note on homogeneous plane continua, Bull. Amer. Math. Soc. vol. 55 (1949) pp. 113-114.

4. H. J. Cohen, Some results concerning homogeneous plane continua, Duke Math. J. vol. 18 (1951) pp. 467-474.

5. F. B. Jones, Certain homogeneous unicoherent indecomposable continua, Proc. Amer. Math. Soc. vol. 2 (1951) pp. 855-859.

6. R. H. Bing, A homogeneous indecomposable plane continuum, Duke Math. J. vol. 15 (1948) pp. 729-742.

7. E. E. Moise, $A$ note on the pseudo-arc, Trans. Amer. Math. Soc. vol. 64 (1949) pp. $57-58$.

8. R. H. Bing and F. B. Jones, Another homogeneous plane continuum (to appear).

9. R. H. Bing, Concerning hereditarily indecomposable continua, Pacific J. Math. vol. 1 (1951) pp. 43-51.

10. L. F. McAuley, On the aposyndetic decomposition of continua, Doctoral Dissertation, University of North Carolina, 1954.

11. F. B. Jones, Concerning non-aposyndetic continua, Amer. J. Math. vol. 70 (1948) pp. 403-413.

12. R. H. Bing, Some characterizations of arcs and simple closed curves, Amer. J. Math. vol. 70 (1948) pp. 497-506.

13. R. L. Moore, Foundations of point-set theory, Amer. Math. Soc. Colloquium Publications, vol. 13, New York, 1932, Theorem 19, p. 340 and Theorem 2, p. 369; and Concerning upper semi-continuous collections of continua, Trans. Amer. Math. Soc. vol. 27 (1925) pp. 416-428, Theorem 22.

14. C. E. Burgess, Some theorems on n-homogeneous continua, Proc. Amer. Math. Soc. vol. 5 (1954) pp. 136-143.

The University of North Carolina 